\title{
Space charged region in GaN and InN nanocolumns investigated by atomic force microscopy
}

\author{
M. Niebelschütz , V. Cimalla , O. Ambacher , T. Machleidt , K.-H. Franke , J. Ristic , J. Grandal , \\ M. A. Sánchez-García, and E. Calleja \\ Institute of Micro- and Nanotechnologies, Technische Universität Ilmenau, P.O. Box 100565, 98684 Ilmenau, Germany \\ Department of Computer Graphics Program, Technische Universität Ilmenau, P.O. Box 100565, 98684 Ilmenau, Germany \\ ISOM-Dept. Ingeniería Electrónica, ETSI Telecomunicación, Universidad Politécnica, 28040 Madrid, Spain
}

\begin{abstract}
High quality InN and GaN nanocolumns of different length and diameter grown by molecular beam epitaxy (MBE) were electrically characterized directly and non-destructively by Atomic Force Microscopy (AFM) as a function of the column diameter. The "exact" column diameter was determined from AFM images by Blind Tip Estimation (BTE) and subsequent image reconstruction in order to avoid artefacts due to the finite AFM tip radius. In GaN, the conductivity rises up to a
\end{abstract}

1 Introduction In recent years group III nitrides have become more and more interesting from both fundamental and applied viewpoints. One reason is the large range of bandgap energies $(0.7-6.2 \mathrm{eV})$ that can be covered by the nitrides and their alloys while keeping its direct character. This wide range is ideal for many optoelectronic applications, like detectors, solar cells, and optical communication systems. One-dimensional nanostructures such as nanowires (NWs) and nanotubes are attractive because of the large surface to volume ratio, which causes a strong dependence on changing environment conditions. With a combination of group III nitrides and one-dimensional nanostructures it is possible to fabricate for example high sensitive chemical sensors. To get these applications at the edge of the technologies it is necessary to have a reliable non-destructive and convenient characterization method.

In a previous publication, the growth of freestanding $\mathrm{GaN}$ and InN nanocolumns by PAMBE on Si(111) was presented. These columns are free of extended "critical" diameter due to a depletion region at the surface of the nanocolumns and remains constant above. In contrast, the electron accumulation at the surface causes decreasing conductivity in $\mathrm{InN}$ nanocolumns with increasing diameter. Thus, the nanocolumn surface acts as the preferential conduction path. These facts prove that there is electron accumulation in as-grown non-polar InN surfaces, according to calculations of the Fermi level pinning in InN. defects and strain as determined by Transmission Electron Microscopy (TEM) and Raman spectroscopy For this reason these samples are optimal to determine fundamental aspects of these materials, in particular, the influence of the surface space charge region on the conductivity. The most common way of the electrical characterization is a structured $\mathrm{Ti} / \mathrm{Au}$ or $\mathrm{Ni} / \mathrm{Au}$ metallization to define contacts to NWs. This procedure requires a transfer of single NW onto a chip, which modifies the original NW and, for example due to radiation damage, influence the surface conductivity. In this paper the electrical characterization of single vertically aligned GaN or InN nanocolumns with varying column diameters without any modification of the sample by Atomic Force Microscopy (AFM) will be demonstrated.

2 Experimental The InN and $\mathrm{GaN}$ nanocolumns are grown by molecular beam epitaxy (MBE) on conductive ntype Si substrates with a resistivity of $0.001 \Omega \mathrm{cm}$ and with average diameters ranging from 40 to $120 \mathrm{~nm}$ and a length of 300 to $1000 \mathrm{~nm}$
Other measurements had shown, 
that there is a good $\mathrm{Si} / \mathrm{InN}$ interface which should result in a good electrical contact. A typical scanning electron micrograph (SEM) of InN nanocolumns is shown in the inset of Fig. 1. For the measurements a NTMDT Solver Pro AFM was used with high resolution silicon cantilevers with a typical tip radius of $35 \mathrm{~nm}$. These tips were coated with 20$35 \mathrm{~nm}$ of Platinum (Pt), Cobalt (Co) or Gold (Au) to achieve good conductivity with a resistance below $1 \mathrm{k} \Omega$ and to realize the electrical contact to the top of the nanocolumn.

In Fig. 1 the experimental setup is shown. Due to the small resistance of $\mathrm{InN}$, a serial resistor to limit the current is used. For the GaN nanocolumns the serial resistor is not necessary (dashed line). To determine the geometries of the columns two measurements were performed. First for the determination of the average length of the nanocolumns a SEM was used, because of the disability of the AFM in this setup to measure the length of the columns. Second, to find a single column and to observe the diameter a tapping mode scan was performed. The result of this measurement is a combination of the "real" diameter and the radius of the AFM tip To get the "exact" diameter two analytical methods, the Blind Tip Estimation (BTE) and a fuzzy-region-growing algorithm, are implemented

In a next step, the AFM tip with the conductive coating is set in contact with a single nanocolumn applying a constant contact force. A voltage is applied between the AFM tip and the n-type $\mathrm{Si}$ and the resulting current through the column is measured. The range of this voltage is -5 to $+5 \mathrm{~V}$ for $\mathrm{GaN}$ and -0.1 to $+0.1 \mathrm{~V}$ for $\mathrm{InN}$.

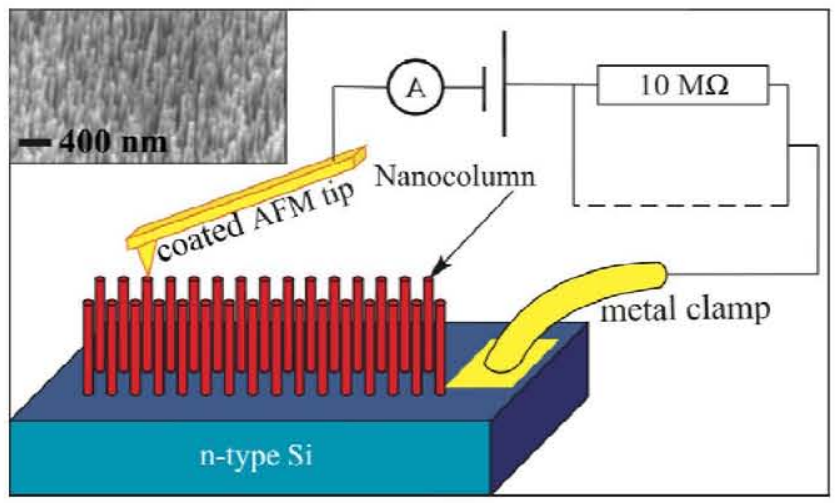

Figure 1 Experimental setup to measure a single nanocolumn with a conductive AFM tip and with a serial resistor of $10 \mathrm{M} \Omega$ or without a serial resistor (dashed line) of $\mathrm{InN}$ and $\mathrm{GaN}$, respectively. Inset: Typical SEM images of InN nanocolumns grown on a $\mathrm{Si}(111)$ substrate

\section{Results and discussion}

3.1 GaN nanocolumns The measurement results of the I-V-characteristics with different AFM tip coating metals and different nanocolumns show a Schottky behaviour with different threshold voltages (inset a Fig. 2). For an ideal contact, the Schottky barrier height is proportional to the difference in work function $w$ between the coating metal $(\mathrm{Au}, \mathrm{Pt}, \mathrm{Co})$ and $\mathrm{GaN}$ surface, which determines the threshold voltage (turn on voltage) $V_{\text {th. }}$. In Fig. 2 the thresh- old voltage for Pt with a work function $w$ of $5.3 \mathrm{eV}$ is higher than for $\mathrm{Au}(w=4.8 \mathrm{eV}) \quad$ however, there is no direct correlation between $w$ and $V_{\text {th }}$ because the difference in work function of only $0.5 \mathrm{eV}$ is faced to difference of $1.8 \mathrm{eV}$ in the threshold voltage. Otherwise Park has shown on $\mathrm{ZnO}$ nanorods that the AFM tip with its small contact radius, in the range of a few $\mathrm{nm}$, has a strong influence on the threshold voltage of a Schottky contact. It was shown that the Schottky barrier height becomes a function of the lateral size for small diodes and this effect influences the electrical measurements by AFM. Thus, the difference of the threshold voltage is determined by the non-classical "nanoSchottky" behaviour and not by the exact work function difference which causes the larger difference.

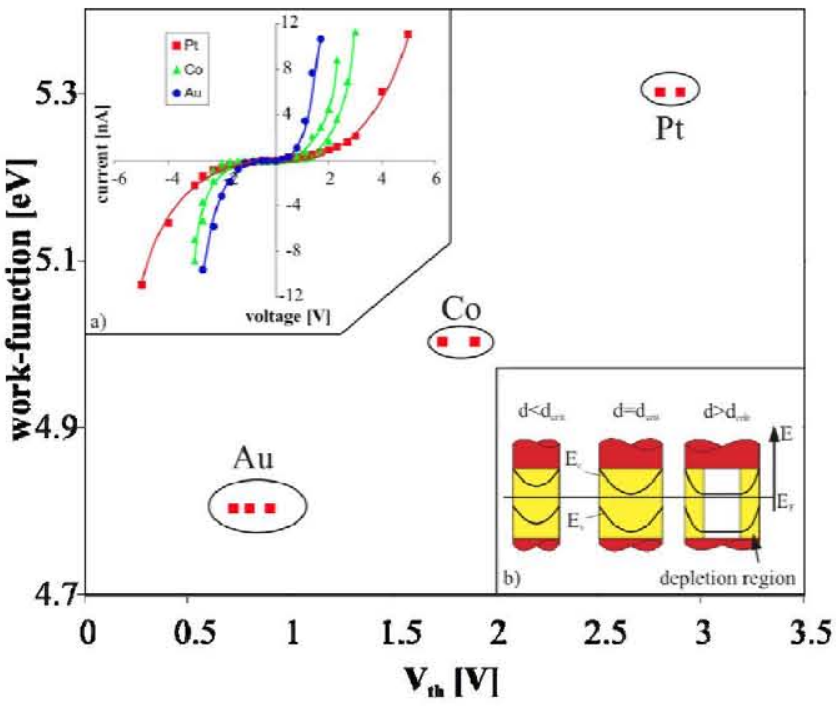

Figure 2 Threshold voltage vs. work function for different coating metals (Pt, $\mathrm{Co}$ and $\mathrm{Au}$ ). Inset a): Current/voltage characteristic of $\mathrm{GaN}$ nanocolumns contacted by different metallized tips (Pt, Co and $\mathrm{Au}$ ). Inset b): Band model of GaN nanowires for different diameters with a depletion region close to the surface (yellow).

The other interesting point is the existence of a "critical" diameter, above which the resistivity of the GaN nanocolumns is constant as in bulk materials (inset b Fig. 2). The calculation of the resistivity is based on the differential resistance of the nanocolumns i.e. the slope of the I-V characteristics at high current is used to characterize the electrical properties of a single GaN nanocolumn. Above the "critical" diameter, the typical measured resistivity of about $11 \Omega \mathrm{cm}$ (see Fig. 3) is in a good agreement with the resistivity values of Calarco The "critical" diameter is the point where the space charge region which is typical for GaN surfaces, spreads out over the whole column diameter. For smaller diameters, the resistivity of the nanocolumns is only affected by the depletion region and rises drastically. In comparison to the results of other groups, the GaN nanocolumns in this work have a comparable resistivity but a smaller critical diameter of about $32 \mathrm{~nm}$, which indicates a smaller depletion region, despite the same expected background doping 
$\left(6.25 \times 10^{17} \mathrm{~cm}^{-3}\right.$ by Calarco et al.

and $5 \times 10^{17} \mathrm{~cm}^{-3}$ in this work, both measured on homogeneous $\mathrm{GaN}$ layers for comparison). This difference can be explained by different preparation methods. The different environments or processing might influence the surface potentials and the width of the depletion region.

3.2 InN nanocolumns InN nanocolumns with similar heights and diameters show a much higher conductivity (Fig. 3). This higher conductivity relates to the tendency of InN to have very high n-type conductivity especially due to an electron accumulation at the surface $[18,19]$ which results in a very small contact resistance. Because of this, there is also dependence on the diameter but in the opposite way as for GaN namely a rise of the resistivity with lager diameter. The trend of the resistivity is not fully clear so far. It seems to be roughly linear with $d(d<100 \mathrm{~nm})$ so it can be assumed that for small diameters the surface conductivity at the sidewalls of the non-polar InN nanocolumn is dominant which fits with Eq. (1), but this linear trend breaks for larger diameters.

$$
\sigma=e \mu\left(n_{b u l k}+\frac{4 N_{S}}{d}\right)
$$

( $e$ and $\mu$ being the electron charge and mobility; $n_{b u l k}$ the background volume carrier density; and $N_{S}$ the surface sheet carrier density). The presence of electron accumulation on non-polar InN surfaces was also demonstrated by Calarco et al. [20] and is proven by simulations and theoretical calculations [21].

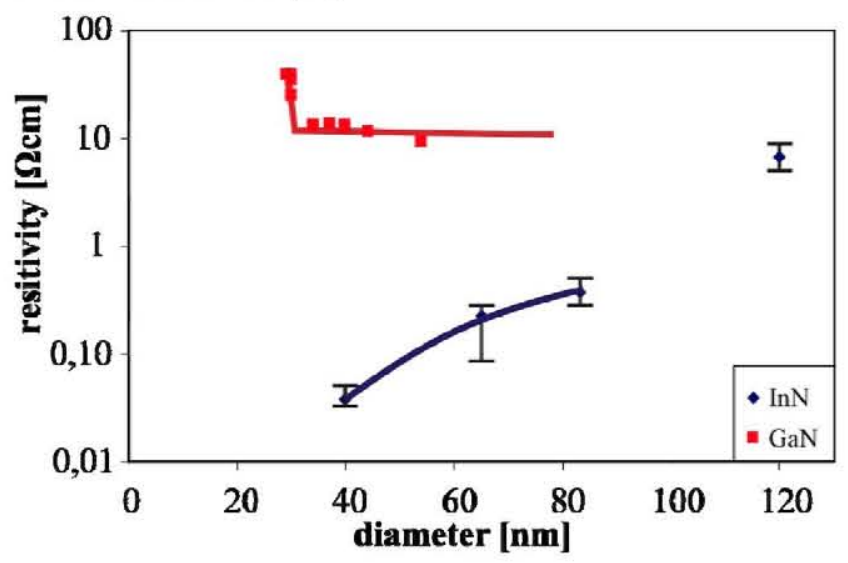

Figure 3 Dependence of the resistivity on the diameter of $\mathrm{GaN}$ and $\mathrm{InN}$ nanocolumns with trend lines as guide to the eye.

4 Conclusion A non-destructive electrical measurement of nanoscale columns by an AFM was demonstrated with the advantage of no further sample preparation, which could influence the results, especially the very sensitive surface accumulation or depletion layer. For GaN nanocolumns a resistivity of about $11 \Omega \mathrm{cm}$ and a critical diameter of about $32 \mathrm{~nm}$ were determined where the depletion region expands to the whole column. In addition, a dependence of the work function difference between AFM tip and
GaN nanocolumn and the threshold voltage was shown, but influenced by the small contact radius. For InN nanocolumns the electrical conduction proceeds in a very different way as in $\mathrm{GaN}$ ones. The conductivity decreases with rising diameter, which confirms the assumption, that there is no surface depletion and the conduction path for small diameters is dominated by an electron accumulation at their lateral non-polar InN surfaces.

Acknowledgements This work is supported by "Deutsche Forschungs Gemeinschaft: SFB 622-A8 Nanopositionier- und Messmaschine", CI148/2-1 "Mech(n)ano", and the Spanish Ministry of Education (MAT2004-2875, NAN04/09109/C04/2, Consolider-CSD 2006-19), the Community of Madrid (GR/MAT/0042/2004 and S-0505/ESP-0200).

\section{References}

S. Nakamura, T. Mukai, and H. M. Seno, Appl. Phys. Lett. 64, 1687 (1994).

S. Nakamura, Science 281, 956 (1998).

S. Strife and H. Morkoc, J. Vac. Sci. Technol. B 10, 1237 (1992).

I. Vurgaftman, J. R. Meyer, and L. R. Ram-Mohan, J. Appl. Phys. 89, 5815 (2001).

D. Zhuang and J. H. Edgar, Mater. Sci. Eng. R. 48, 1 (2005).

V. Dobrokhotov et al., J. Appl. Phys. 99, 104302 (2006).

L. Cerutti, J. Ristić, S. Fernández-Garrido, E. Calleja, A. Trampert K. H. Ploog, S. Lazic, and J. M. Calleja, Appl. Phys. Lett. 88, 213114 (2006)

M. A. Sánchez-García, J. Grandal, E. Calleja, S. Lazic, J. M. Calleja, and A. Trampert, phys. stat. sol. (b) 243, 1490 (2006).

T. Stoica, R. Meijers, R. Calarco, T. Richter, and H. Lüth, J. Cryst. Growth 290, 241(2006).

E. Calleja, M. A. Sanchez-Garcia, F. J. Sanchez, F. Calle, F. B. Naranjo, E. Munoz, U. Jahn, and K. Ploog, Phys. Rev. B 62, 16826 (2000).

J. Ristic, M. A. Sanchez-Garcia, E. Calleja, J. SanchezParamo, J. M. Calleja, U. Jahn, and K. H. Ploog, phys. stat. sol. (a) 192, 60 (2002).

H. Romanus, J. Schadewald, V. Cimalla, M. Niebelschütz, T. Machleidt, K.-H. Franke, L. Spiess, and O. Ambacher, Microelectron. Eng. 84, 528 (2007).

T. Machleidt, R. Kästner, K.-H.Franke, in: Reconstruction and geometric assessment of AFM tips, in: Nanoscale Calibration Standards and Methods, edited by G. Wilkening, L. Koenders (Wiley-VCH, 2005).

C. Freyhardt et al., Exp. Phys. 6, 873 (1992).

S. P. Muraka, Metallization - Theory and Practice for VLSI and ULSI, pp. 250 (1993).

J. G. Park et al., Appl. Phys. Lett. 81, 4625 (2002)

R. Calarco et al., Nano Lett. 5, 5 (2005).

V. Cimalla et al., phys. stat. sol. (a) 203, 59 (2006)

V. Cimalla, G. Ecke, M. Niebelschütz, O. Ambacher, R. Goldhahn, H. Lu, and W. J. Schaff, phys. stat. sol. (c) 2, 2254 (2005).

R. Calarco and M. Marso, Appl. Phys. A 87, 499 (2007).

D. Segev and C. G. Van de Walle, Surface Sci. Lett. 601, L15 (2007). 